\title{
Systematic Reviews: Significance of Search Strategy
}

\section{P.A.S.H. Perera ${ }^{1}$}

\begin{abstract}
Systematic reviews are yet to evolve in Sri Lanka despite its widespread use in developed countries. A systematic review (SR) is a literature review which aims to address a specific research question and follow scientific methodology with a predefined protocol. It is conducted by a team of reviewers. In most of the developed countries where evidence-based practice is desired, findings of SRs have been used as an aid for developing policy and guidelines in social sciences and healthcare. The procedure of SRs is a set of scientific methods; systematic, comprehensive and rigorous. Searching for ALL relevant literature for inclusion in a SR is an integral part of the procedure which requires expertise and skills in information retrieval. With recent advances in standards, guidelines and methodology of SRs, the construction of search strategy to locate all relevant literature/studies has become a challenging task for review authors. Need for support from information specialist has been recognized in this exercise, thus creating a significant role for library professionals to play in the search process of systematic reviews. The aim of this article is to provide the local LIS community with an introduction to SRs and to discuss briefly the significance of the search strategy in locating the studies in a SR process.
\end{abstract}

Keywords: Best Evidence, Systematic Literature Search, Conducting Systematic Reviews, Search Strategy, Evidence Synthesis, Systematic Review Methodology

${ }^{1}$ Senior Assistant Librarian, University of Peradeniya, Email: sriper@pdn.ac.lk

(iD) http://orcid.org/0000-0002-3713-3304

(c) (9)
(C)
4.0 International License. 


\section{Introduction}

Literature Reviews are studies that are commonly carried out by researchers and scholars in Sri Lanka like many other countries. Nevertheless, Systematic Reviews (SRs) appear to be less understood by researchers, not only in Sri Lanka but elsewhere as well. In Sri Lanka Systematic Reviews are yet to evolve whereas it is widespread and gaining popularity in the developed world. While literature on SR is widely available through various resources on the internet and in print format, the purpose of this article is to provide an insight to Systematic Reviews and make the Library and Information Science (LIS) professionals in Sri Lanka (who are not familiar with SRs) aware of its characteristics. The article aims to describe briefly what SRs are all about, the procedure of how to conduct SRs and also focuses on the element of 'search strategy' of SR procedure which is of much importance and relevance to library professionals. Much of the content of the article is based on author's experience through conducting SRs and the deliberations at SR Training Program at the Health Sciences Library System (HSLS) of School of Medicine of University of Pittsburgh, USA which is conducted on a regular basis with sponsorship of the Medical Library Association, USA.

The idea of evidence-based practice (EBP) is not new and has been in existence worldwide over several decades. Interest in evidence-based practice has been growing across the globe in recent times, especially in the social and health sciences. Its widespread acceptance as the most desirable practice for many scenarios is much evident in healthcare. In the context of EBP, systematic reviews are considered as one of the key sources of information in finding the much needed 'best evidence' on any given topic.

"Healthcare decision makers including clinicians and other healthcare providers increasingly turn to systematic reviews for reliable, evidence-based comparisons of health interventions"(Eden, Levit, Berg \& Morton, 2011, p.17).

Systematic reviews have been in use for varying purposes in social sciences for decades. Today in most of the developed countries especially in the United State of America and in Europe SRs are being increasingly used in decision making processes such as developing policy and guidelines to support practice. In addition, findings of SRs have been used as guides to 
direct future research efforts in many fields of sciences. Particularly in health sciences where high quality evidence and guidance are of vital importance in formulating clinical practice guidelines and improving health care decision making, SRs are considered to be the most reliable source of best evidence.

A scrutiny through history of SRs reveals its roots from as far back as 1891(Petticrew and Roberts, 2006). In many instances, though the term "Systematic Reviews" has been used to refer to literature reviews, most of the processes have not been really systematic. During 1960s and 1970s Systematic Review methods underwent a transformation (Glass, 1976). This followed by a wide spread use of SRs by 1980s particularly in the USA and UK, resulted in a rapid increase in the number of publications of SRs in health care (Petticrew and Roberts, 2006). Opening of the UK Cochrane Center in 1993 and establishment of NHS Center for Reviews and Dissemination in 1994 were among many developments in the UK, to support preparation, maintaining and dissemination of SRs (Chalmers, Hedges, \& Cooper, 2002; Petticrewand Roberts, 2006). In 1993 Cochrane Collaboration was established in the USA (Chalmers, Hedges, \& Cooper, 2002; Lefebvre, Glanville, Wieland, Coles \& Weightman, 2013) with the aim of collecting and summarizing the best evidence from research to help make informed decisions for improved healthcare.

Scoping search (electronically) done by the author for articles on systematic reviews carried out in Sri Lanka/by Sri Lankan authors/about Sri Lanka yielded only four articles (Rajapakse, Griffiths \& Christensen, 2013, Pearson, Zwi, Rouse, Fernando, Buckley \& McDuie-Ra, 2015, Lunyera, Mohottige, Isenburg, Jeuland, Patel \& Stanifer, 2015,Ranasinghe, Jayawardena \& Katulanda, 2015). All four of these studies have been conducted with foreign collaborations.

\section{What is a Systematic Review?}

SRs are Research studies on 'Research' where findings of existing studies are used as raw data."Subjects" of SRs are individual primary studies: primary studies to SRs are what individual participants are to primary studies (Perera, 2016). 
As the term implies, Systematic Reviews would adhere to a set of scientific methods; pre-planned with a well-specified protocol. Categorically, they are literature reviews which would address a clearly formulated research question; aim to provide an objective and comprehensive summary of a large body of literature with a view to finding the most reliable evidence to help answer the question (Glasziou, 2001; Perera, 2016). In this task, the procedure includes several steps: identify and select articles/publications (of studies) relevant to the research question; critically assess those selected studies and then collect and analyze the data from those studies; as the final step, combine (narratively or statistically) the findings of those individual studies (included in the review), and prepare a comprehensive summary depicting the conclusion (Perera, 2016).

Different terminology has been used for SRs by researchers, such as 'systematic literature reviews', 'evidence synthesis' and 'research synthesis'. However the widely used term at present is 'systematic reviews'(Petticrew and Roberts, 2006).

The difference between systematic reviews and other types of literature reviews has been well defined. Traditional/narrative reviews discuss and aim to provide a broad over view of the evidence pertaining to a given question/topic. In contrast, as explained above, SRs adhere to an explicit and pre-specified methodology and aim to answer a specific question or test a specific hypothesis. Systematic Reviews differ from traditional/narrative reviews especially in methodology with regard to: Study protocol, Search strategy, Study selection criteria, Assessment of study quality and Data synthesis.

A meta-analysis goes hand-in hand with systematic reviews. Meta-analysis is the use of statistical techniques to integrate the results of included studies in a SR. However, it is not necessary for a SR to include a meta-analysis and SRs could be presented alone or in combination with meta-analysis (Manchikanti, 2009). There are times that quantitative analysis (metaanalysis) is not appropriate or possible in SRs depending on the nature of the studies included in the review (Lau, 1997). A good example is when the pool of data extracted from included studies in a SR is heterogeneous. 
Conducting a systematic review is more time consuming, require more effort and information resources than other types of reviews.

\section{Importance of Systematic Reviews}

In developed economies, the decision makers appear to rely heavily on SRs for determining public policy, clinical practice guidelines and informed health care choices to ensure that their decisions are based on best available research evidence. However this can be a difficult process. Research information in the form of journal articles, books, electronic resources, conference proceedings, reports and theses etc. are generated exponentially in some fields by various types of studies. These studies could be of varying designs such as Randomized Control Trials, Controlled Trials, Cohort Studies, prospective/retrospective studies or Observational Studies etc. Therefore, integrating evidence of such a body of literature to find the best evidence is a difficult task. In such a situation, according to Petticrew and Roberts (2006) a well-conducted SR can be considered as the most authoritative source of information to use to find the best evidence. Figure-1 illustrates the hierarchy of health/medical research information resources.

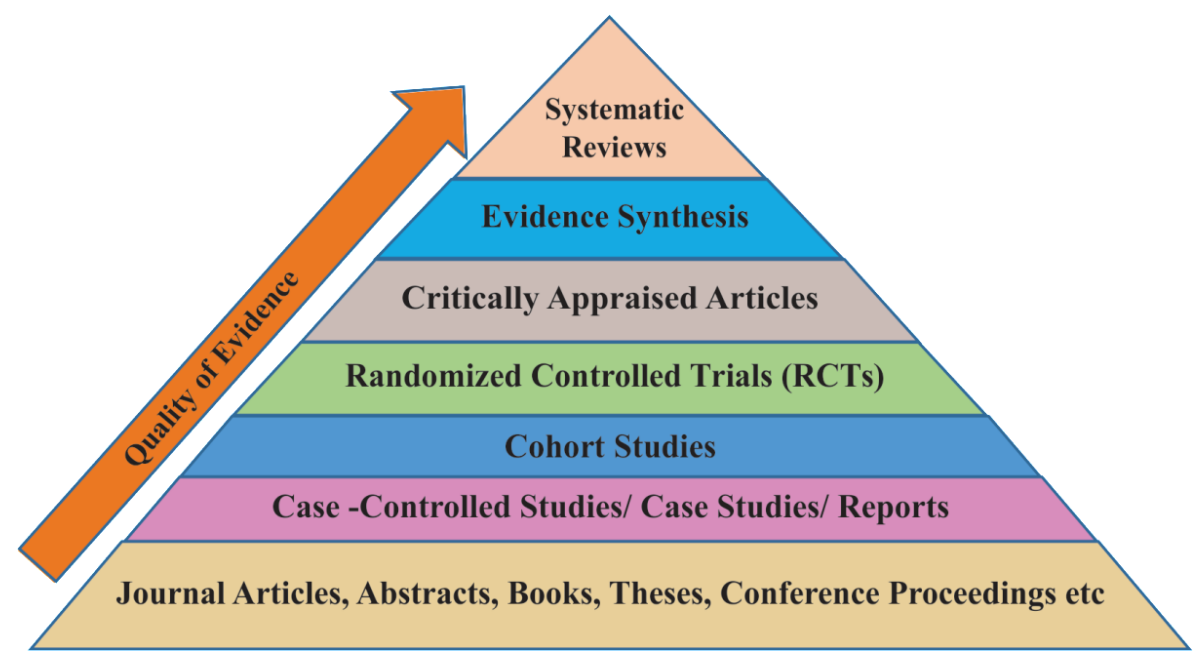

Figure 1: Finding the Best Evidence

(Adapted from: EBM Pyramid and EBM Page Generator, copyright 2006 Trustees of Dartmouth College and Yale University)

Findings of single studies cannot be considered as definitive and conclusions cannot be made upon such evidence in any given situation due to limitations 
inherent in many studies. It is known that some studies have to be repeated several times; sometimes by different research groups in different study settings, using different methodologies. Each of these studies may be subject to various biases, differ in quality of methodology and design, dependent on time and context. Often, the results of these multiple studies are diverse; sometimes reach conflicting conclusions; making it difficult to figure out what exactly is reliable. Thus making a conclusion as to which evidence to be used as a basis for practice and policy decision may be in a dilemma. In this context, combining and synthesizing results/evidence from different studies and integrating it to generate new data would have the potential to provide evidence with enhanced power (Glasziou, 2001) give more reliable and definite interpretation of the entire body of evidence on a given topic. Hence, as Petticrew and Roberts (2006) points out, in addressing a particular issue/question specially if there is an uncertainty about the answer, SRs could be of great value as a means of reviewing all the available evidence relevant to the question, to clarify what works and what does not about the issue in the question (Figure:2).Further, SRs are considered the best, least biased and most logical and scientific way to collect, evaluate, interpret and integrate the research evidence. On the other hand SRs can be considered as a scientific tool; a means of dealing with the information overload by distilling a large amount of research information into a manageable form (Petticrew and Roberts, 2006; Perera, 2016). It implies that 'systematic reviews' unfold science of synthesizing research.

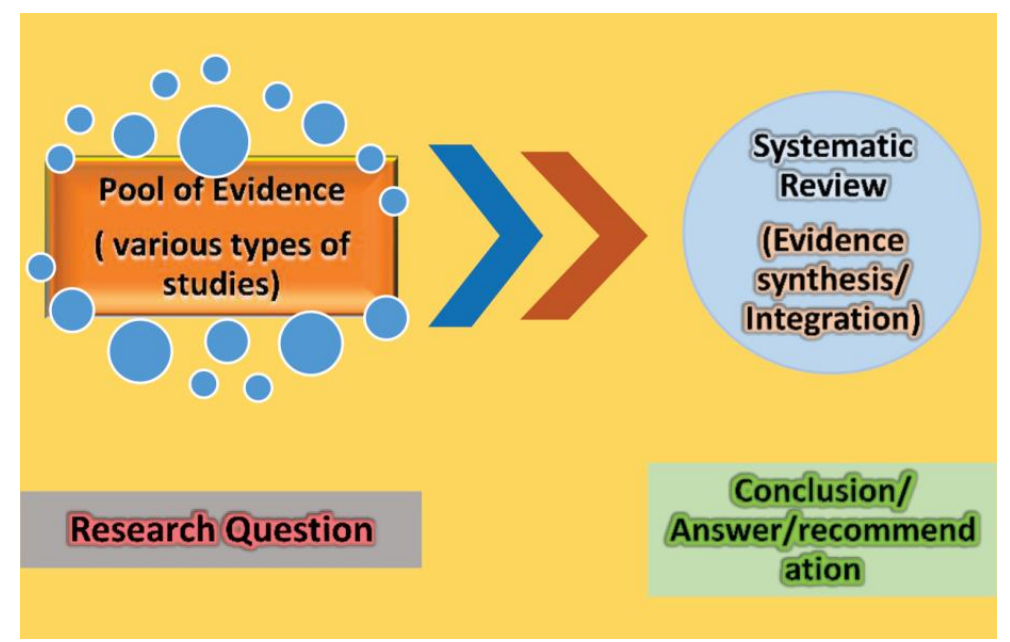

Figure 2 : Importance of Systematic Reviews (Perera, 2016) 
Systematic reviews are widely used to synthesize evidence in studies which examine effectiveness of interventions in various scenarios and also in studies requiring to find an answers to questions about etiology or causes of problems (Petticrew and Roberts, 2006). Some examples of questions addressed in published Systematic Reviews include:

1. Characteristics of non-fatal self-poisoning in Sri Lanka (Rajapakse, Griffiths \& Christensen, 2013).

2. A Systematic Review of Water Fluoridation - UKbased study(McDonagh, et al., 2000)

3. CKD of Uncertain Etiology: A Systematic Review - Sri Lanka based study (Lunyera, Mohottige, Isenburg, Jeuland, Patel, \& Stanifer, 2015)

4. Role of Video Games in Improving Health-Related Outcomes: A Systematic Review - USA based study (Primack, et al., 2012)

\section{Need for a Systematic Review}

It is always better considering whether a systematic review is needed or not before deciding on it. There may be a several number of reasons for considering a systematic review to be conducted. When there is an uncertainty about the effectiveness of a policy or service, where there has been some literature on the issue that does not help solve the problem may be an example of a situation requiring a SR (Petticrew and Roberts, 2006). Similarly in questions about interventions (social or health care), treatments, prevention, diagnosis, prognosis or etiology or questions about general opinion of people where many research has been carried out but important questions remain unanswered would be another candidate for a SR (Petticrewand Roberts, 2006). On topics where a gap in knowledge is identified thus requiring evidence synthesis or where prioritizing of funds is needed and requiring a definite choice to be made, are two more examples of situations in which SRs would be of help. Sometimes SRs are investigator led, with topics identified from an area of practice or research interest; whatever the motivation for undertaking a SR may be, the preparation and conduct should be rigorous (Centre for Reviews and Dissemination, University of York, 2009). 


\section{Procedure of Systematic Reviews}

There are several protocols available to describe the procedure of SR. Presented in this article is based on the training program on SR for medical librarians at HSLS, School of Medicine, University of Pittsburgh, USA.

Ensuring reliability and quality of a SR is a major concern. To address this issue, SRs should be conducted and results should be reported according to specifications that have been established through various standards and guidelines. In clinical, biomedical and healthcare research the methodology for SR has been well established and guidelines/standards to assist reviewers, have been produced by Cochrane collaboration and IOM-Institute of Medicine (name changed to Health and Medical Division in March 2015) of National Academies of USA. Cochrane guidelines (Higgins \& Green, 2009) and CRD's Guidance (Centre for Reviews and Dissemination, University of York, 2009) are such documents which are being used in health science research world-wide. However, in social sciences and multidisciplinary research SR methodology has not been used as much as in healthcare nor do any guidelines exist to assist reviewers in these fields. However, to help reviewers in these fields in adapting the Cochrane guidelines, several research centers and academic institutions (example: Social Care Institute for Excellence, UK) have taken steps to set their own frameworks for systematic reviews and have produced various toolkits and specialized guidelines (Jesson, Matheson \& Lacey, 2011). Reporting results of SRs is an important aspect of conducting SRs and adhering to PRISMA guidelines (Moher, Liberati, Tetzlaff\& Altman, 2009) is widely accepted.

In general, before embarking on a SR, it is necessary to search through literature to check whether there already exists or ongoing a SR on the same topic thus justifying another systematic review. In case where an existing SR is identified on the same question/topic, then it is important to assess it for its quality, design and setting to determine its suitability for the intended purpose in policy and practice. If it is of adequate quality and relevance then a new SR on the same question may not be carried out. In disciplines/fields where research is rapidly evolving and information is generating fast, then an update of the existing review may be needed. 
In this paper only a summary description of each step of the procedure will be given in the interest of limiting the length of the publication. A properly conducted systematic review explicitly aims at limiting bias of all types in each step of the procedure. Reviewers need to be comprehensive, paying attention to all the technicalities in each step along the review process. Steps in conducting a systematic review:

1. Assess need; assemble the review team with required expertise It is recommended that a minimum of two reviewers are included in the team which comprises of expertise in SR methodology, information retrieval and subject specialty relevant to the research question.

2. Define the review question clearly; break it into sub questions using the format PICOS (Participants, Intervention or exposure, Comparison, Outcomes and Study design).

3. Create the protocol (Plan) for the study; follow standards and guidelines. ( CRD, IOM)

The protocol should define and specify all the technical details pertaining to each step of the process, including 'Study Inclusion Criteria'(inclusion/exclusion) for the review which specifies the types of studies (by design) to be searched/located in order to answer there view question. Study types could be from Randomized Control Trials, Controlled trials, Prospective cohort studies or Observational studies to Case-control studies etc.

Protocol also should outline the 'search strategy' to locate articles/studies, methods of managing retrieved references and data extraction. Method for assessing/appraising of studies for their quality in terms of possible biases in the methodology and heterogeneity of data should be clearly described in the protocol.

4. Search for literature: strive to locate ALL relevant published and unpublished studies. Search Strategy is constructed using search terms based on the concepts of PICOS and should include searching through All relevant databases and other sources including greyliterature (conference proceedings, trial registers, theses and dissertations, reports of organizations, government publications etc..). 
5. Select studies for the review which meet "Study Inclusion Criteria (SIC)". The criteria is drawn normally upon the desired scope, relevance to the research question, population characteristics, intervention details, language, study type and publication type of individual studies. The SIC may vary from review to review depending on the review question. It is recommended that selection of studies is conducted in two steps: review by title/abstract first and then review by reading through the full-text article. Each study should be checked for its compliance with the SIC. According to IOM standards 'study selection' should be undertaken by at least two independent reviewers with a third person available for conflicts resolution. To ensure transparency, it is important to keep a $\log$ of excluded studies (with reasons) and to have whole process documented. (electronically is preferred)

6. Data Extraction: collect data from above selected individual studies that includes study characteristics such as population, age, gender etc. and findings(outcomes)of those studies. Using a data extraction form makes this exercise easy and systematic. Here again it is recommended to consider data extraction by more than one observer.

7. Quality Assessment of those selected studies: in technical terms this is referred to as 'critical appraisal'. The main objective of this exercise is to determine whether the quality of the study is adequate enough in methodology and measure of outcomes to answer the research question. Reviewers should assess whether the study is representative of the wider population and also whether individual studies are subjected to any elements of bias that might affect the final interpretation of the results of the systematic review. A number of guides and tools are available for use in this process. However, using simple checklists or guides is desirable to assess strength and weakness of each study against its various characteristics ranging from study design to outcomes and further to reporting/presenting. Considering assessment by more than one reviewer is recommended.

8. Synthesis of evidence drawn from included studies: based on the decision whether it would be a narrative synthesis or a quantitative analysis (meta-analysis) of data whichever is appropriate. This 
depends on the nature of the pool of data extracted from selected studies where a narrative synthesis is recommended if the data are heterogeneous.

Integrate results of only studies which are most methodologically sound. A narrative synthesis would systematically combine/compare the findings/evidence of those individual studies. A meta-analysis would combine data from individual studies to draw up a definite measure of effect on the intervention in question.

9. Write a comprehensive summary of the review: make conclusions based only on studies of highest quality.

10. Document and archive search strategy, review data and all the relevant materials.

11. Disseminate findings: write the report according to PRISMA guidelines: the findings of the SR can be presented as an article in a scholarly journal.

12. Update the review as needed.

\section{Searching for Information: Significance of the Search Strategy}

A systematic review requires a rigorous and exhaustive search to locate the relevant literature. Thus, identifying the research evidence/studies for the review is an integral part of the SR procedure. This process, being very systematic, comprehensive and transparent the reviewers need to strive to finding all relevant studies published as well as unpublished (ie., those which are not in the public domain), where possible, in order to provide the best evidence that helps answer the research question. A comprehensive search is best achieved by using a carefully developed search protocol consisting of a range of methods and approaches (electronic and manual), to locate relevant literature through multiple resources. Therefore SRs are dependent on availability of access to appropriate databases, the nature/quality of those databases and also the comprehensiveness of the search. The identification of relevant studies for a SR has been considered as the most challenging exercise for systematic reviewers, requiring information literacy skills, which are often not possessed by them (Pettigrew and Roberts, 2006). Working with an information scientist who has the expertise in 'searching' would ensure that the most effective approach is used; the best search strategy is formulated by identifying suitable search terms/keywords and by using the most up-to-date and appropriate resources that can be accessed. The search protocol consisting of choosing databases, term harvesting and search strategy construction should be clearly defined in the study protocol."The 
development of a search strategy is an iterative process: one attempt will rarely produce the final strategy"(Jesson, Matheson \& Lacey, 2011).

In some journals it is a requirement for a systematic review to have the electronic search strategy peer-reviewed by an independent librarian or information scientist who has the expertise in it and by using appropriate checklists. The quality of a search strategy/protocol is dependent on its effectiveness, transparency and reproducibility. Further in meeting its mission to be comprehensive, SRs require additional searching other than database searching. This process includes searching for grey literature; handsearching of key journals; cited/citing referencing which are known as 'snowballing'/'pearl growing'(Glasziou, 2001;Petticrewand Roberts, 2006 \& Lefebvre et al, 2013). Grey literature is best defined as:

"That which is produced on all levels of government, academics, business and industry in print and electronic formats, but which is not controlled by commercial publishers." (Fourth International Conference on Grey Literature, 1999; Haig and Dozier, 2003)

With recent developments, revision and implementation of quality improvement measures on standards of methodology of SRs, the quality of information retrieval aspect has become challenging for researchers (Lefebvre et al., 2013). At present, the standards developed by the US Institute of Medicine specifies the requirement of having a librarian/information specialist in the review team (Eden, et al., 2011).

As a conclusion remark it is worth mentioning that keeping abreast with research methodologies including systematic reviews, emerging technologies such as textual analysis, semantic analysis, text mining etc. and ever expanding resources of information as well as acquiring required skills and expertise to support researchers in their respective disciplines must be an essential focus of professional development for librarians especially in academia. 


\section{References}

Centre for Reviews and Dissemination, University of York, (2009). Systematic Reviews: CRD's Guidance for Undertaking Reviews in Health Care.CRD, University of York. York. Retrieved from: Website: www.yps-publishing.co.uk

Chalmers,I., Hedges, L.V. \& Cooper, H.A. (2002).A brief history of research synthesis. Evaluation and the Health Professions, 25, 12-37.

Eden, J., Levit, L, Berg, A, Morton, S. (Eds). (2011). Finding What Works in Health Care: Standards for Systematic Reviews. Washington DC: Institute of Medicine: National Academies. DOI: 10.17226/13059

Fourth International Conference on Grey Literature.(1999). New frontiers in grey literature: GL'99 proceedings, Oct 4-5; Washington,D.C, GreyNet. Available from: http://www.greynet.org/opensiglerepository.html

Glass, G.V.(1976). Primary, secondary and meta-analysis of research. Educational Research, 5, 3-8

Glasziou, P., Irwig, L., Bain, C.\& Colditz, G. (2001). Systematic reviews in health care: a practical guide. Cambridge, U.K., Cambridge University Press Retrieved from: http://site.ebrary.com/lib/ubdlib/Doc?id=10006795

Haig, A.\& Dozier, M. (2003). BEME (Best Evidence Medical Education) Guide No 3: Systematic searching for evidence in medical education. Edinburgh, UK. NHS Education for Scotland.

Higgins, J.P.T.\&Green, S. (Eds). (2011). Cochrane Handbook for Systematic Reviews of Interventions, Version 5.1.0, London, The Cochrane Collaboration, Available from: www.handbook.cochrane.org.

Jesson, J.K., Matheson, L. \&Lacey, F.M. (2011). Doing your literature review: traditional and systematic techniques. London : Sage publications.

McDonagh, M., Whiting, P., Wilson, P., Sutton, A., Chestnutt, I., Cooper, J.,Kleijnen, J. (2000) Systematic review of water fluoridation. British Medical Journal, 321, 855-9. 
Manchikanti, L., Benyamin, R., Helm, S. \& Hirsh, J.A. (2009). EvidencedBased Medicine, Systematic Reviews and Guidelines in Interventional Pain management: Part 3: Systematic Reviews and Meta-Analyses of randomized Trials. Pain Physician, 12, 35-72.

Moher,D., Liberati,A., Tetzlaff ,J.\& Altman, D.G. (2009). Preferred Reporting Items for Systematic Reviews and Meta-Analyses: the PRISMA statement (2009). Journal of Clinical Epidemiology,62 ,1006-1012.

Lau, J., Ioannidis, J.P. \&Schmid, C.H. Quantitative synthesis in systematic reviews. (1997). Annals of Internal Medicine, 127, 820-826.

Lefebvre, C., Glanville, J., Wieland, L. S., Coles, B.\&Weightman, A. L. (2013). Methodological developments in searching forstudies for systematic reviews: past, present and future? Systematic Reviews, 2(78), Retrieved from:

http://www.systematicreviewsjournal.com/content/2/1/78

Lunyera, J., Mohottige, D., Isenburg, M., Von, Jeuland, M., Patel, U.D. \&Stanifer, J. W. (2015). CKD of Uncertain Etiology: A Systematic Review. Clinical Journal of the American Society of Nephrology, 11, Doi : 10.2215/CJN.07500715.

Pearson, M., Zwi, A. B., Rouse, A. K., Fernando.,Buckley, N. A.\&McDuieRa, D. (2015). Taking Stock - What Is Known About Suicide in Sri Lanka: A Systematic Review of Diverse Literature Crisis (2015), 35, 90-101. DOI: 10.1027/0227-5910/a000244.

Perera, S. (2016). Systematic Reviews: Science of Synthesizing. Research Magazine of University of Peradeniya: Hantana Vision, 2(2). 8-10.

Retrieved from: https://www.pdn.ac.lk/uop/inrc/ResearchMagazine.php

Petticrew, M. \& Roberts, H.(2006). Systematic Reviews in the Social Sciences: a practical guide. Oxford, Blakwell.

Primack, B.A., Carroll, M.V., McNamara, M., Klem, M. L., King, B., \&Nayak, S. (2012). Role of Video Games in Improving HealthRelated Outcomes, A Systematic Review. American Journal Preventive Medicine, 42(6), 630-638, doi:10.1016/j.amepre.2012.02.023. 
Rajapakse,T, Griffiths, K.M. \& Christensen, H. (2013). Characteristics of non-fatal self-poisoning in Sri Lanka: a systematic review. $B M C$ Public Health, 13, 331.

Retrieved from: http://www.biomedcentral.com/1471-2458/13/331.

Ranasinghe, P., Jayawardena, R. \&Katulanda, P. (2015). The facts, figures, and reality of the diabetes epidemic in Sri Lanka: a systematic review. International Journal of Diabetes in Developing Countries, 35(4), 501-513. DOI:10.1007/s13410-015-0326-z 